\title{
Democracia y Participación ciudadana en Chile. Un análisis desde la Teoría del Derecho ${ }^{1}$
}

J. Ignacio Núñez Leiva ${ }^{2}$

\section{RESUMEN}

El presente trabajo revisa cuatro variables o fenómenos que distinguen el estado actual de la participación ciudadana en Chile: una constante y casi irreductible desconfianza en el sistema político, un creciente privatismo, una considerable divergencia semántica entre el concepto de Democracia operativo en los Estados de Derecho contemporáneos y los presentes en el discurso popular y, una neutralidad en el juicio moral respecto de las formas políticas en general y en particular acerca de la Democracia. Propone estrategias teóricas para revertir dichas características, especialmente las dos últimas, promoviendo un papel protagónico a hombres y mujeres de Derecho, específicamente el de ser agentes comunicadores de las principales transformaciones acontecidas en la conceptualización de la Constitución y que ha impactado a las teorías del Derecho yla Democracia.

\section{PALABRAS CLAVE}

Neoconstitucionalismo, Democracia, Participación, Constitucionalismo contemporáneo.

\section{PÓRTICO}

He sido invitado para que efectúe un análisis de la participación ciudadana en Chile, objetivo que intentaré cumplir. Sin embargo, hago presente que mi trabajo estará marcado por un sesgo insoslayable. En efecto, mi formación como hombre de Derecho hace que, inevitablemente, cualquier tipo de análisis que realice sea desde la perspectiva de un Cientista Jurídico. Por ello, la ponencia que en esta oportunidad expondré lleva por título: Análisis de la Democracia y la participación desde la Teoría del Derecho.

\section{DEMOCRACIA $Y$ PARTICIPACIÓN}

Diversos estudios se han efectuado en torno al conflictivo binomio democracia - participación. Entre ellos, por sus calidad, rigurosidad y solidez metodológicas destacan: el informe del Programa de las Naciones Unidas para el Desarrollo del año 2004 (PNUD 2004) titulado La Democracia en América Latina, el informe Participación Ciudadana en Políticas Públicas de la coalición Más voces para el fortalecimiento de la Democracia, de 2005 (Más Voces 2005) y los periódicos informes de la

${ }^{1}$ Ponencia presentada en el seminario La situación actual de la Democracia, organizado por la Unlversidad San Sebastián sede Concepción el 29 de Abril de 2009 y en los III Encuentros Regionales de Derecho Público, organizados por la Universidad de las Américas, sede Viña del mar los días 13 y 14 de agosto de 2009.

${ }^{2}$ Abogado. Licenciado en Derecho, Pontificia Universidad Católica de Chile. Diplomado en Metodologías de Investigación e intervención social, Universidad Alberto Hurtado. Diplomado en Derechos Humanos, Universidad Católica del Uruguay. Especialista en Constitucionalismo y Garantismo, Universidad de Castilla La Mancha, España. Magíster $\mathcal{O}$ en Derecho Público, Pontificia Universidad Católica de Chile. Doctorando en Derecho, Universidad de Castilla La Mancha, España. Secretario de Estudios y Docente de la Facultad de Derecho, Universidad San Sebastián, Santiago de Chile. Profesor de las Facultades de Derecho de las Universidades Andres Bello y Alberto Hurtado. E-mail: jinunez@uc.cl 
Corporación Latinobarómetro. ${ }^{3}$ Nuestro análisis se desarrollará a partir de ellos.

Cada uno de los estudios mencionados, incorporan un amplio número de interesantes variables que servirían para analizar el problema que hoy nos convoca. Sin embargo, su análisis requeriría de más tiempo del que disponemos. Por ello, hemos escogido intencionadamente aquellas que son funcionales a la descripción de los fenómenos y/o procesos que me interesa destacar en esta oportunidad.

Tales procesos son los siguientes: En primer lugar una constante y casi irreductible desconfianza en el sistema político, en segundo lugar un creciente privatismo, en tercero la existencia de una considerable divergencia semántica entre el concepto de Democracia operativo en los Estados de Derecho contemporáneos y los presentes en el discurso popular y, en cuarto lugar, una neutralidad en el juicio moral respecto de las formas políticas en general y en particular acerca de la Democracia. A continuación revisaremos los antecedentes que justifican dicho diagnóstico.

\section{Desconflanza, privatismo, divergencias semánticas y neutralidad.}

Diversos indicadores revelan la presencia de un estado permanente de desconfianza en el sistema político, particularmente en los órganos de naturaleza representativa, lo cual se manifiesta en diversas esferas. Por ejemplo, según el informe 2008 de Latinobarómetro, en Chile, el $71 \%$ de los encuestados piensa que el Estado está gobernado por unos pocos para proteger los intereses de los poderosos ${ }^{4}$, solo el $31 \%$ evalúa como bueno o muy bueno el desempeño del Congreso Nacional y únicamente el $31 \%$ de los consultados manifiesta tener algún grado de confianza en dicha institución. ${ }^{5}$

Conjuntamente con lo anterior, se puede apreciar la paulatina consolidación de un privatismo, entendiendo a este como una inclinación por la satisfacción de los intereses privados por sobre los públicos o sociales conjuntamente con la tendencia a no establecer lazos de asociatividad con terceros, si que, ni lo uno ni lo otro, implique actos contrarios a Derecho. En tal sentido, son reveladores los siguientes índices: según el informe 2005 de la Coalición Más Voces, un $56 \%$ de los chilenos encuestados no participa en ningún tipo de forma asociativa destinada a colaborar para resolver problemas colectivos. De entre las personas que $s i$ participan, los conglomerados con mayor número de integrantes son de naturaleza religiosa 0 de indole deportiva. ${ }^{6}$ El mismo estudio indica que un $65 \%$ de los encuestados manifiesta trabajar nunca o casi nuca en conjunto con otros para resolver sus problemas y que el $82 \%$ reconoce no haber concurrido nunca en conjunto con otros ante una autoridad para requerir la solución de un problema.

Por su parte, cuando se consulta a las personas por el significado que para ellos tiene el concepto Democracia, aún ante preguntas con respuesta cerrada, los resultados demuestran una clara pluralidad de significados. Por ejemplo, según el informe 2005 de Latinobarómetro, Democracia significa para los sudamericanos: Libertades civiles o individuales en un $38 \%$ de los casos, igualdad y justicia en un $11 \%$, Derecho a voto en un $6 \%$, paz y unidad en $5 \%$, Estado de Derecho en un $2 \%$, etc.

Tal divergencia semántica no es apreciable sólo en el discurso social, sino también en la doctrina. Conocidas son las variadas concepciones acerca de la democracia, que abarcan desde su entendimiento como un simple procedimiento de adopción de decisiones colectivas, hasta su comprensión como una especial forma de vida. Por otra parte, en sistemas con restauración democrática reciente, como lo son la mayoría de los sudamericanos, es menester tener presente que la forma política denominada Democracia suele representar para quienes experimentaron épocas dictatoriales, precisamente aquellos valores $\mathrm{y} / 0$

${ }^{3}$ Especialmente el informe 2008.

${ }^{4}$ Latinobarómetro (2008) p. 82.

${ }^{5}$ Idem. p. 85.

${ }^{6}$ Más Voces (2005) p. 4.

'Latinobarómetro (2005) p. 40. 
derechos que fueron conculcados en tales periodos. Ello puede ayudamos a comprender, en parte, la existencia de esta divergencia semántica.

Por último, como se adelantó, también es posible identificar una cierta neutralidad hacia las formas políticas en general y en particular en torno a la Democracia. Para este propósito resultan sumamente claros los resultados obtenidos al hacer escoger a los encuestados entre prosperidad económica y democracia: según el informe 2008 de latinobarómetro, en Chile, un $49 \%$ de las personas no ve obstáculos en que un gobierno sea no democrático en tanto resuelva sus problemas económicos. ${ }^{8}$ Esta proporción, desde hace años, no ha variado mucho. En efecto, según el informe PNUD del año 2004, hacia el año 2002 casi la mitad (48,1 por ciento) de los encuestados que expresaban valorar democracia por sobre cualquier otro régimen, prefería igualmente el desarrollo económico a la democracia, y un porcentaje semejante (44,9 por ciento) que decía preferir la democracia estaba dispuesto a apoyar a un gobierno autoritario si éste resolvía los problemas económicos de su país. ${ }^{9}$ El mismo informe señala que ante la variable "Están de acuerdo con que el presidente vaya más allá de las leyes" un $58,1 \%$ de los encuestados de la muestra total, respondió afirmativamente.

Sin lugar a dudas, las cuatro tendencias y fenómenos revisados influyen en las bajas tasas de participación y en funcionamiento de la Democracia. La desconfianza en el sistema democrático desmotiva la participación ciudadana, el privatismo lo deslegitima, las divergencias semánticas favorecen evaluaciones negativas acerca de su funcionamiento pues lo evalúan en base a expectativas que no necesariamente pude cumplir, y la neutralidad moral acerca de su vigencia abona un terreno fértil para sus críticos. Ante este escenario la pregunta ineludible es la siguiente ¿Qué hacer? Como ya adelanté, la respuesta que propongo viene teńida por el sesgo de un cientista jurídico.
Respecto del primer fenómeno, la desconfianza, algo ya se está haciendo.

La reforma constitucional del año 2005 marcó el primer paso constitucionalizar los principios de probidad y transparencia, y la reciente entrada en vigencia de la ley de transparencia avanza otro tanto en el mismo sentido. Corresponde ahora estar atentos a su funcionamiento y a la promoción del accountability.

Sobre el segundo de los factores, el privatismo, mucho tiene que decir la educación en Derechos Humanos, y especialmente, en palabras de Häbermas, la generación de un patriotismo constitucional que destaque la condición de todos los seres humanos de titulares de la misma dignidad, y por tanto de las mismas virtudes y miserias, fomentando, de paso, las relaciones de colaboración ysolidaridad.

En ambos casos, la ciencia jurídica, tiene mucho de decir. Sin embargo, a mi juicio, es respecto de los dos factores restantes donde el papel de los hombres y mujeres de Derecho resulta crucial e insustituible. A mi juicio, la divergencia semántica en torno al concepto de democracia y la neutralidad moral hacia ella mucho tienen que ver con un generalizado desconocimiento acerca de su funcionamiento y con la anacrónica vigencia de un concepto ya superado de democracia, es decir, con la visión positivista o paleopositivista del Estado de Derecho y de la Democracia. Cuestión que abordaremos a continuación.

\section{Estado Legislativo de Derecho y Democracia.}

El contenido del concepto "Democracia" se ha ido perfilando durante la historia. No solo en la esfera de su justificación, sino también en cuanto su rol y perfiles al interior del Estado de Derecho. Respecto de este último punto resulta crucial identificar la profunda transformación que para la noción de Democracia ha significado el advenimiento del Estado Constitucional de Derecho en remplazo del

\footnotetext{
${ }^{8}$ Según el mismo informe, el promedio sudamericano ante esta pregunta es el 53\%.

'PNUD (2004) p. 137.
} 
Estado Legislativo de Derecho. Sobretodo en aquellos Estados herederos de la tradición revolucionaria francesa, estructurados en base a los principios sentados por ella.

Para darnos cuenta de lo profundo de esta transformación revisaremos brevemente los perfiles de la Democracia en el Estado legislativo de Derecho, encarnado en el Estado modelado por la revolución francesa, y los confines de la Democracia en la actualidad, al alero del Estado Constitucional de Derecho.

La revolución francesa, vanguardia, al menos conceptual, del Estado de Derecho europeo y cuna de la formulación más difundida de la separación de poderes, condujo a un diseño institucional, notablemente influido por las ideas de Montesqieu y Rousseau, en el cual la fuente protagónica del Derecho y la política fue la ley. ${ }^{10}$

El espacio intelectual de 1789 - señala Prieto Sanchís - se encontraba definido por la intención de generar un orden nuevo que marcara la profunda ruptura con el antiguo régimen. Ello puede apreciarse en su documento fundacional: la Declaración de derechos del hombre y del ciudadano que contiene un diseño institucional basado en el iusnaturalismo racionalista, en principios "simples e incontestables" que se exponen como verdades evidentes de las ciencias, con un sentido antihistórico que no implica ninguna clase de confirmación o recepción de elementos del pasado y con derechos naturales subjetivos que no dejan espacio a ninguna ley natural objetiva, pues fue precisamente esta última la que sirvió de legitimación al régimen derrocado. ${ }^{11}$

En este contexto - continúa el filósofo del Derecho -en el racionalismo del siglo XVIII, algunos, influidos por el pensamiento de Rousseau ${ }^{12}$, creyeron que, la justicia del Derecho, dependía no tanto del respeto a algunos principios inmutables y sustantivos, no tanto de la limitación al poder soberano desde una instancia externa, sino de las cualidades internas del propio legislador. No era, en definitiva, el carácter absoluto e ilimitado del poder lo que lo convertía en peligroso, sino su ejercicio por un sujeto malicioso o incompetente. Al respecto cabe destacar que el preámbulo de la declaración habla de principios "sencillos e incontestables" que deben orientar los actos de los poderes público, en definitiva, principios evidentes. $Y$ se inicia, justamente, afirmando que "la ignorancia, la negligencia, o el desprecio de los Derechos Humanos son las causa de calamidades públicas y la corrupción de los gobiernos".

Por su parte, apoyando el juicio de Prieto Sanchís, Zagrebelsky, señala que, en esencia, la revolución francesa no fue una causa contra el absolutismo monárquico, lo fue solo contra la monarquía. ${ }^{13}$

Así, con la reasignación de la soberanía al pueblo, su nuevo titular la hereda sin transformar en profundidad las cualidades que al poder absoluto se solían atribuir. El nuevo modelo de legitimidad no repara en los límites al poder, sino en que sea el pueblo quien legisla. Y si los antiguos legisladores no estaban sometidos a límite alguno, tampoco debía estarlo el pueblo ni la manifestación de su voluntad soberana. ${ }^{14}$ De alli que ninguna otra norma - incluyendo la Constitución - pudiera limitar la voluntad general contenida en la ley.

En abono de lo anterior el ya citado catedrático español trae a colación el artículo cuarto de la Declaración en cuanto señala que los límites a los derechos son los propios derechos, pero que es la ley quien determina cuales son los derechos. ${ }^{15}$ Lo cual se ve ratificado por la afirmación de Fioravanti en orden a que para el pensamiento francés de la

${ }^{10}$ VILE (2007) p. 198.

${ }^{11}$ PRIETO (2003) p.54.

${ }^{12} \mathrm{En}$ El contrato social se lee: “Es contrario a la naturaleza del cuerpo político que el soberano se imponga una ley que no puede infringir (...) no hay ni puede haber ninguna especia de ley fundamental obligatoria para el conjunto del pueblo, ni siquiera el contrato social"

${ }^{13}$ ZAGREBELSKY (2007) p.25.

${ }^{14}$ Según el artículo 60 de la declaración "La ley es expresión de la voluntad de la comunidad"

${ }^{15} \mathrm{El}$ mencionado artículo reza: La libertad política consiste en poder hacer todo aquello que no cause perjuicio a los demás. El ejercicio de los derechos naturales de cada hombre, no tiene otros límites que los necesarios para garantizar a cualquier otro hombre el libre ejercicio de los mismos derechos; y estos límites sólo pueden ser determinados por la ley. 
época el legislador no puede lesionar los derechos individuales porque este es necesariamente justo. ${ }^{16}$

El pensamiento de Rousseau no solo influye en la caracterización de la ley y por tanto en la teoría del Derecho que surge con la revolución. Impacta además en la teoría constitucional y en la teoría de la democracia atemperando-e incluso modulando la formulación de la teoría de separación de poderes sistematizada por Montesqieu.

El poder judicial, prontamente fue perfilado como un mero aplicador de la ley y se intentó reducir al mínimo posible la eventualidad de que alterase el contenido de esta. Ya en 1791 se le prohibió de forma expresa cualquier tipo de revisión de la ley promulgada, sea anulatoria o suspensiva, imperativo que permanece hasta la actualidad. Este hecho da cuenta de una nota distintiva de la teoría de separación de poderes revolucionaria de raíz francesa: la absoluta ausencia de controles heterónomos de Derecho activos o pasivos sobre la labor legislativa y en particular sobre las leyes vigentes. La soberania de la ley no define solo sus propiedades cuasi míticas, sino también la teoria constitucional.

Por su parte, el concepto de democracia que surge de la revolución es, paradójicamente, restrictivo y materialmente ilimitado. Restrictivo puesto que la participación del pueblo en el gobierno mediante la regla de la mayoría se universalizará en tanto los actualmente partícipes consientan la inclusión de más participantes en el universo electoral, mediante, claro está, decisiones de mayoría. $Y$ materialmente ilimitado en tanto no contempla normas 0 valores supremos inviolables 0 irreductibles, cotos vedados ni ámbitos de 10 indecidible. La propia declaración ha señalado que existen derechos naturales, sin enumerarlos ni precisarlos y ha dispuesto también que la imposición de cualquier limite a esos derechos es resorte de la ley.

Esta forma de ver las cosas marcará claramente la posición relativa de las fuentes del Derecho y de los poderes del Estado. Para la tradición revolucionaria francesa la Constitución será un documento que da vida a un programa político específico: el de la soberanía popular. Pero en caso alguno puede llegar a ser un límite a la soberanía del pueblo ni a la ley, ello explica - entre otras cosas - el tardío y particular sistema galo de justiciaconstitucional.

Por su parte, en este contexto, la separación de poderes se inclinará por reconocer un rol preponderante al órgano legislativo por sobre los otros poderes.

\section{Constitucionalismo y Democracia.}

A partir del siglo XX surge y se consolida una nueva forma de ver las cosas y las relaciones entre los factores ya descritos - se trata del constitucionalismo -corriente de pensamiento que se distingue por su afán por describir y destacar los logros de la constitucionalización, es decir, de aquel proceso causado por una revitalizada Constitución que ha ocasionado una profunda modificación de los sistemas jurídicos y que deviene en la creación del Estado Constitucional de Derecho.

El cambio mencionado se produce a partir de la introducción de conceptos como el valor normativo de la Constitución y la vinculación de la jurisdicción a los derechos fundamentales. ${ }^{17}$ Ello es el corolario de la fusión de dos tradiciones, la de las constituciones materiales; aquellas que presentan un denso contenido sustantivo integrado por normas de diferente valoración (valores, principios, derechos, directrices) y las constituciones garantizadas, caracterizadas porque su protección - efectividad se encomienda a jueces 0 , si se prefiere, en su sistema existen normas secundarias, de organización y procedimiento, destinadas a depurar o sancionar toda infracción de las normas sustantivas o relativas a derechos.

Así la Constitución se convierte en límite y norma directiva fundamental. Frente al rousseauniano

${ }^{16}$ FIORAVANTI (2007) p. 73.

${ }^{17}$ JULIO ( 2007) p. 148. 
poder constituyente que nunca termina de constituirse - nos dice Prieto-y que desemboca en la superioridad del parlamento y de su ley - lógica básica de los sistemas de raíz francesa - el poder constituyente toma cuerpo en un texto jurídico supremo, válido, vigente y garantizado. ${ }^{18}$

El resultado de esta combinación, señala con contundencia Ferrajoli, es un nuevo modelo de Derecho y Democracia: el Estado Constitucional de Derecho, que es fruto de un verdadero cambio de paradigma respecto al modelo paleopositivista del Estado Legislativo de Derecho ${ }^{19}$ y en el cual, siguiendo la clasificación de Guastini, la constitución es fuente del Derecho en un triple sentido: primero, por contener normas, segundo, por disponer parámetros sustantivos y procedimentales para abrogar y/o invalidar normas inferiores preconstitucionales e invalidar normas posteriores que la contravengan, y tercero - siendo quizás esto lo más destacable - por entenderse que las normas constitucionales son idóneas para disciplinar directamente no sólo la organización estatal y las relaciones entre el Estado y las personas, sino también las que ocurran ente estas últimas, y son por tanto susceptibles de ser aplicadas por cualquier juez y no solo por el juez constitucional. ${ }^{20}$

El valor normativo de la Constitución como concepto fundante de los neoconstitucionalismos produce una serie de consecuencias relevantes, entre ellas, Robert Alexy destaca: la sustitución de las reglas por los principios como elementos protagónicos del Derecho, la necesariamente consecuente utilización de la técnica de la ponderación en desmedro de la subsunción para la aplicación de las normas, la omnipresencia de la Constitución en lugar de la independencia del derecho infraconstitucional y el fortalecimiento del poder de la judicatura en perjuicio de las facultades del legislador. ${ }^{21}$
Todo lo anterior claramente altera el paradigma decimonónico del Estado de Derecho y afecta las bases del sistema de fuentes elaborado a su amparo, las de su teoría constitucional y del Derecho, y su concepto de Democracia.

Con respecto al sistema de fuentes, la Constitución irrumpe como fuente del Derecho en el sentido pleno de la expresión, es decir, como origen inmediato de derechos y obligaciones, y no solo como fuente de las fuentes. ${ }^{22}$ Ello altera la relación constitución-ley.

En lo relativo a la teoria constitucional, las relaciones reciprocas entre las funciones del Estado se alteran sustantivamente. El esquema decimonónico en el cual el legislador se encuentra investido con la capacidad de representar a la soberanía y por tanto invocar su inviolabilidad, resulta alterado por la aparición de una jurisdicción, ya sea ordinaria o específicamente constitucional, capaz de oponerse al contenido sustancial de sus decisiones, sea impidiendo que se conviertan en Derecho vigente o suprimiéndolas del sistema jurídico con efecto general o particular según sea el caso, encargada de un continuo adecuamiento de la legislación a la Constitución mediante la tutela de las exigencia de la justicia sustancial en cada caso concreto y que se erige como el principal agente racionalizador del sistema jurídico. ${ }^{23}$ Una función muy difícil de encuadrar en la formulación tripartita de la separación de poderes, e incluso en ciertos casos contramayoritaria.

Por su parte, la democracia operativa dentro del paradigma paleopositivista - citando a Ferrajoli - se ha convertido en Democracia constitucional. Este es un modelo que no se limita a programar solo las formas de producción del Derecho mediante norma procedimentales sino que además programa sus contenidos. Está constituido por normas metalegales que disciplinan la máxima expresión

\footnotetext{
${ }^{18}$ PRIETO (2007) p. 214.

${ }^{19}$ FERRAJOLI (2007) 2007, p. 71.

${ }^{20}$ GUASTINI (2007) p.22.

${ }^{21}$ ALEXY (1994) p. 160.

${ }^{22}$ RUBIO (1979) p. 62.

${ }^{23}$ POZZOLO (1998) p. 342.
} 
de las mayorías contingentes presentes en el proceso de generación de las leyes. En síntesis, la Constitución es aquel Derecho por sobre el derecho y opera como una convención sobre el coto vedado ${ }^{24} 0$ acerca de lo que es indecidible para cualquier mayoría. ${ }^{25}$

La constitucionalización rígida de los derechos fundamentales- nos sugiere el célebre jurista de Florencia - impone obligaciones y prohibiciones a los poderes públicos $y$ ha insertado en la democracia una dimensión sustancial. Si las normas formales de la Constitución, esto es aquellas que disciplinan la organización de los poderes públicos, aseguran la dimensión formal de la democracia política, aquellas que establecen los principios y Derechos Fundamentales garantizan lo que se puede denominar democracia sustancial, y se refieren a qué no puede ser decidido o debe ser decidido por toda mayoría, vinculando toda la legislación bajo pena de invalidez a los derechos, principios y valores fundamentales. Ello cambia también la relación entre Derecho y política, pues ya no es el primero quien se subordina a la segunda, sino a la inversa. ${ }^{26}$

\section{Reflexiones finales:}

Concluyendo nuestro análisis, resta únicamente ensayaruna especie de síntesis.

En primer lugar, hemos propuesto que a partir de los diversos estudios que nos describen la situación actual de la democracia podemos destacar la presencia de cuatro importantes fenómenos; desconfianza en el sistema político, especialmente en las instituciones representativas, un creciente privatismo, divergencias semánticas respecto del concepto Democracia y neutralidad en el juicio moral respecto de las formas políticas.

En segundo lugar, se ha destacado que tales fenómenos se vinculan a la Ciencia Jurídica y en virtud de aquello, dicha rama del conocimiento puede colaborar en la consolidación de la Democracia.

$Y$, en tercer lugar, hemos señalado que a la hora enfrentar los problemas que surgen de las divergencias semánticas acerca del concepto de Democracia y la neutralidad en el juicio moral de sobre las formas políticas, la Ciencia Jurídica debe asumir un rol fundamental, este consiste en difundir y educar en las transformaciones que ha expenimentado el diseño institucional del Estado de Derecho, describiendo de manera adecuada el Estado Constitucional de Derecho y el paradigma actual de la Democracia: la Democracia Constitucional, solo de esta manera se logrará que los juicios y discursos que se emiten sobre ella la evalúen en su real dimensión, se comprenda debidamente su significado $y$, en definitiva se avance en la superación de lo que algunos llaman la crisis de la Democracia.

${ }^{24}$ GARZÓN (1.989) p. 209.

${ }^{25}$ FERRANOLI (1998) p.858.

${ }^{26}$ FERRAJOLI (2008) p. 32. 


\section{BIBLIOGRAFÍA}

1. ALEXY, Robert, El concepto y la validez del Derecho, Gedisa, Barcelona, 1994.

2. CORPORACIÓN LATINOBARÓMETRO, Informe anual año 2005, Santiago de Chile, 2005.

3. CORPORACIÓN LATINOBARÓMETRO, Informe anual año 2008, Santiago de Chile, 2008.

4. FERRAJOLI, Luigi, Derecho y razón. Teoría del garantísmo penal. Madrid, Trotta, 1998.

5. FERRAJOLI, Luigi, La democracia constitucional, en Luigi Ferrajoli: Democracia y Garantísmo, CARBONELL, Miguel, (Editor) Trotta, Madrid, 2008.

6. FERRAJOLI, Luigi, Sobre los derechos fundamentales, en Teoría del neoconstitucionalismo, ensayos escogidos, CARBONELL, Miguel (Editor), Trotta, Madrid, 2007.

7. FIORAVANTI, Mauricio, Los derechos fundamentales. Apuntes sobre historia de las constituciones, Trotta, Madrid, 2007.

8. GARZÓNVALDÉS, Ernesto (1989):Algo más acerca del coto vedado, en Doxa, $N^{\circ} 6$.

9. GUASTINI, Ricardo, Sobre el concepto de Constitución, en Sobre los derechos fundamentales, en Teoria del neoconstitucionalismo, ensayos escogidos, CARBONELL, Miguel (Editor), Trotta, Madrid, 2007.

10. JULIO ESTRADA, Alexei, Los tribunales constitucionales y la eficacia entre particulares de los derechos fundamentales, en Teoría del neoconstitucionalismo, ensayos escogidos, CARBONELL, Miguel (Editor), Trotta, Madrid, 2007.

11. POZZOLO, Sussana (1998): Neoconstitucionalismo y especificidad de la interpretación constitucional, en Doxa, $N^{0} 21$.

12. PRIETO SANCHÍS, Luis, El constitucionalismo de los derechos, en Teoría del neoconstitucionalismo, ensayos escogidos, CARBONELL, Miguel (Editor), Trotta, Madrid, 2007.

13. PRIETO SANCHÍS, Luis, Justicia constitucional y derechos fundamentales, Trotta, Madrid, 2003, p.54.

14. PROGRAMA DE LAS NACIONES UNIDAS PARA EL DESARROLLO (PUND), La Democracia en América Latina, BuenosAires, Aguilar, 2004. 